\title{
MODELING AND SIMULATION OF TEMPERATURE DISTRIBUTION IN LASER CUTTING OF TI-ALLOY SHEET
}

\begin{tabular}{|l|l|}
\hline Shivani Pandey & Arun Kumar Pandey \\
Mechanical Engineering Department & Mechanical Engineering Department \\
Jaypee University of Engineering and & Jaypee University of Engineering and \\
Technology & Technology \\
Guna, India & Guna, India \\
pandey.shivani25@gmail.com & arunpandey76@gmail.com \\
\hline
\end{tabular}

\begin{abstract}
Laser cutting process is thermal energy based advanced machining process. Due to thermal nature of the process, some defects related to thermal such as heat affected zone, oxide layers and micro-cracks may be observed at the cut edges of laser cut specimens. And also, the thermal conductivity as well as thermal diffusivity of Tialloy is very low, so the temperature is concentrated at the localized area in cut edges. The conventional cutting of Tialloys is difficult due to its properties such as low thermal conductivity, low thermal diffusivity and low elastic modulus. Laser cutting may be used for the cutting of these alloys. In this paper, the temperature distribution during the laser cutting of Ti-alloy sheet has been simulated by using finite element based ANSYS software. For the simulation, moving heat source has been considered. The simulation results show that the maximum temperature is obtained just below the laser beam. As the laser beam moves, there is reduction in the temperature in the moving direction of laser beam. The maximum temperature is found in very narrow area just below to the laser beam due to very low thermal conductivity of Ti-alloys.
\end{abstract}

Keyword- Laser cutting, Ti-alloy, Modeling and Simulation, Temperature Distribution, Finite Element Method

\section{INTRODUCTION}

Laser beam machining is the process in which the laser is used to cut the materials by focusing it on the surface of the material [1]. The laser cutting of Ti and its alloys have many applications in various fields such as aerospace food, chemical etc. $\mathrm{Ti}$ and its alloys are difficult to cut by laser as they have low thermal conductivity and high chemical reactivity [2]. Also high temperature gradient arises near the cut edges that cause high temperature and thermal stresses near it. The physical properties of $\mathrm{Ti}$ and its alloys are good that make it more suitable for use, such as at high temperature they have high stiffness and high strength. Also they have high strength to weight ratio and relatively high melting point [3]. Ti-6Al-4V is the most commonly used alloy. It has a chemical composition of $6 \%$ aluminium, $4 \%$ vanadium, $0.25 \%$ (maximum) iron, $0.2 \%$ (maximum) oxygen, and the remainder titanium. It is significantly stronger than commercially pure titanium while having the same stiffness and thermal properties (excluding thermal conductivity, which is about $60 \%$ lower in Grade-5 $\mathrm{Ti}$ than in commercially pure Ti). Among its many advantages, it is heat treatable. This grade is an excellent combination of strength, corrosion resistance, weld and fabricability.

The laser is kept at a constant distance from the work surface of the material and the material is removed by melting and vaporization process [1]. As the laser beam moves forward on the work surface of the material, the surface cools down due to radial heat conduction and convection process from the surface. This phenomenon leads the temperature variation at the cut surface. The temperature gradient attains high values at the region next to the cut surface which ultimately leads to the decay of the temperature rapidly once the laser is passed over the surface. Thermal stresses are generated due to high temperature and high cooling rates during the cutting process. The laser beam machining is an advanced machining process. The main advantage of the process is that it can cut complex shapes and structure very easily with precision and high dimensional accuracy. Laser cutting is a thermal process so there will be thermal stresses generated around the cut edges [4]. The schematic laser cutting process is shown in Fig.1. The laser cutting process requires high power density. Also cutting speed and pulse width are the important factors that control the laser cutting of difficult-to-laser cut materials like $\mathrm{Ti}$ and its alloys. This leads to the heating, melting, vaporization and re-solidification. So the understanding of the temporal variation of the temperature is important to obtain the required results.

Many research studies have been carried out to examine the laser cutting process and the effect of different process parameters on different quality characteristics in laser cutting process. Arif and Yilbas [5] compared the temperature distribution and thermal stresses for three different substrate materials i.e. for steel, Inconel 625 and Ti-6Al-4V. They found out that temperature decay sharply at the vicinity of the cut edges and the equivalent stresses attains high values in this region. The thermal stress is more profound in Inconel 625 followed by steel and Ti-6Al-4V. Laser inert gas cutting of Inconel 718 for thickness $1 \mathrm{~mm}$ and $2 \mathrm{~mm}$ was simulated by finite element analysis by Nyon et al [6]. 


\section{ELK Asia Pacific Journals - Special Issue \\ ISBN: 978-81-930411-4-7}

They showed that kerf width increases with an increase in laser power and decreases with the increase in cutting speed. Thermal analysis showed that the stress is maximum at the central region because the material is constraint to expand at both the ends. Sharma et al. [7] used finite element analysis to explain that the high

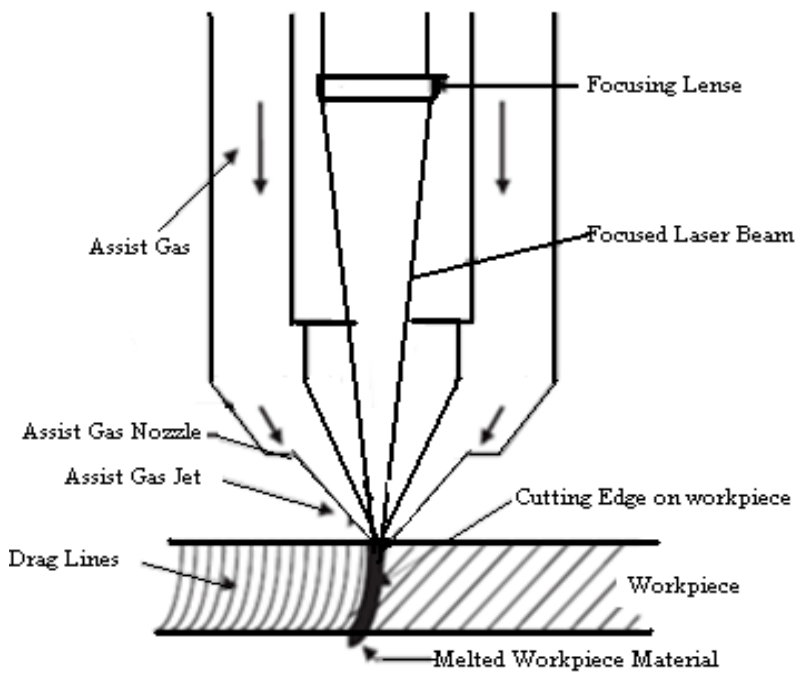

Fig.1. Schematic of laser beam cutting [3]

temperature gradient exists at the laser irradiated spot which results high thermal stresses at the cut section. They also found that the maximum temperature obtained during laser cutting is reduced with increasing laser scanning velocity. Sundar et al. [8] performed the laser decoating of the TiN from tungsten carbide using an excimer laser. The finite element results showed that the temperature of the TiN coating exceeds the boiling point and the maximum stresses formed during the process is comparable to the yield strength of TiN.

Finite element models were developed by Shalahim et al. [9] to stimulate the laser micromachining of acrylic sheet with different process parameters. Akhtar et al. [10] examined the rectangular geometry on the thermal stress field in the cutting section. It was found that the temperature and the stress fields are affected by the size of the rectangular cut geometry. Akhtar [11] performed the laser cutting of thick section circular mild steel blanks. Finite element method was used to predict the thermal and stress fields developed at the cut section. It is found that laser cutting of thick steel blanks results in substantial conduction loss from the cutting zone, which results in high-temperature gradients and large stress levels in the cutting section. Laser straight cutting of alumina tiles is carried out by Yilbas et al [12]. Temperature and thermal stress fields developed in the cutting section are modeled and predicted through the finite element method. It is found that the residual stress predicted agrees with that obtained from the X-ray diffraction technique. Striation patterns formed at the kerf surface have shallow depths because of the low thermal conductivity and high melting temperature of the workpiece. Akarapu et al. [13] developed a mathematical model that describes the ablation phenomena, the thermal stress evolution, and the failure process that occur during laser cutting or forming operations on ceramic plates. Nisar et al. [14] performed the finite element modeling of the cutting process of glass by using different beam geometries, to simulate the transient effects of the moving beam and predict thermal fields and stress distribution. It was found that in comparison to other beam geometries, the triangular-forward beam at the leading edge and triangular-reverse and circular beam geometry at the trailing edge produces lower tensile stresses and hence minimizes the cut path deviation. Yilbas et al. [15] investigated laser straight cutting of zirconia tiles using finite element codes. Their model has shown that the temperature variation along the workpiece thickness is gradual resulting insmall temperature difference between top and bottom surface along the cutting edge. Von Mises stress attains the high values in the region near the cutting edges. Shuja and Yilbas [16] used finite element method to predict temperature and stress distribution in the irradiated region. The laser scanning velocity is assumed to be constant with thermal properties to be temperature dependent. Qui et al. [17] developed an asymmetrical mathematical model to predict temperature and thermal stress for pulsed $\mathrm{Nd}$ : YAG laser heating of 2024 Aluminium alloy. The Gaussian beam geometry was considered and the results showed that the plastic damage occurs in the top layer of the plate where the residual stresses are tensile. Gross et al. [18] simulated the laser-gas-assisted cutting process. They predicted the thermal stresses in the substrate material. The two-beam laser processing of nonmetallic materials was considered and modeled by Shalupaev et al. [19]. They used the finite element method to predict the thermo elastic fields in the heated material. Pandey and Dubey [20] performed the simultaneous optimization of kerf taper and surface roughness in the laser cutting of titanium alloy sheet (Grade-5). They presented optimal solutions and improvements in different quality characteristics of laser cutting. A hybrid approach of neural network and fuzzy logic has been applied by Pandey and Dubey [21] for the prediction of laser cutting process behavior of $\mathrm{Ti}$ alloy sheet (Ti-6Al-4V). They compared the predicted results with the experimental data and found it appropriate.

The aim of this paper is to develop a finite element model by using ANSYS finite element code to determine the temperature distribution during the laser cutting of the titanium alloy sheet. The model is based on 
the transient heat transfer equation and the heat losses are by radial conduction and convection process.

\section{HEAT TRANSFER ANALYSIS}

Before the heat transfer analysis is done, there is need for the modeling of the heat source. Various beam shapes have been used for different applications. Some commonly used beam shapes are Gaussian, rectangle, circular triangular etc. The Gaussian energy distribution is mostly commonly used mode for laser cutting because a very small diameter can be easily focussed which results in high power density [7]. The following equation defines the laser beam intensity on the surface;

$I_{s}=I_{0} \exp \left(-\frac{2 r^{2}}{w_{0}^{2}}\right)$

Where, $I_{s}$ is the laser beam intensity, $I_{0}$ is the laser intensity at $(0,0), \mathrm{r}$ is defined as the distance from the center of the beam, and $w_{0}$ is the radius at which the profile of the laser beam is shown in Fig. 2. Amplitude is $1 / \mathrm{e}$ of its value on the axis.

The heat transfer analysis of the laser beam intensity is done by using two dimensional transient heat equations. The spatial and temporal distribution, $\mathrm{T}(\mathrm{x}, \mathrm{y}$, t) satisfies the following differential equation for two dimensional heat conduction in a domain D [2]. The governing equation for the model is based on the Fourier law heat conduction and is given as;

$\frac{\partial}{\partial x}\left[k(T) \frac{\partial T}{\partial x}\right]+\frac{\partial}{\partial y}\left[k(T) \frac{\partial T}{\partial y}\right]+Q_{\text {int }}=\rho c(T) \frac{\partial T}{\partial t}$

Where, $k(\mathrm{~T})$ thermal conductivity as a function of temperature $(\mathrm{W} / \mathrm{mK}), \rho$ is the material density $\left(\mathrm{kg} / \mathrm{cm}^{3}\right)$, $\mathrm{c}(\mathrm{T})$ is specific heat as a function of temperature $(\mathrm{J} / \mathrm{kg} \mathrm{K})$ and $\mathrm{Q}_{\text {int }}$ is the heat generation rate $\left(\mathrm{W} / \mathrm{m}^{3}\right)$. The variable properties that are used in the simulation process are given in Table I. For solving the governing equation the initial boundary of the material is assumed to be at reference temperature $\mathrm{T}_{0}$, so

$T(x, y, 0)=T_{0}$ for $(x, y) \in D$

In addition at a distance far away from the surface in $x-y$ plane the temperature becomes equal to the reference temperature. This will yields the boundary condition of

$T(0, y, t)=T$ for $t>0$
At the free surface $S_{1}$, a convective boundary is assumed, therefore the corresponding boundary conditions are as follows:

$q_{0}=h\left(T_{\text {ext }}-T\right)$

on the boundary $S_{l}$ for $(\mathrm{x}, \mathrm{y}) \in S_{l}$ and $\mathrm{t}>0$.

Where, $\mathrm{T}_{\text {ext }}$ is the external temperature and $\mathrm{h}$ is the heat transfer coefficient $\left(\mathrm{W} / \mathrm{m}^{2} \mathrm{~K}\right)$.

\section{FINITE ELEMENT MODELING}

The finite element modeling is a mathematical modeling that is basically used to find out the approximate solution of the given differential equation. It uses integral formulations rather than differential equations as in the finite difference method to create a system of algebraic equations [5]. The governing equation of the structural problem is the principle of the virtual work. A virtual (very small) change of the internal strain energy $(\delta U)$ must be equal to an identical change in external work due to the applied loads $(\delta \mathrm{V})$. Considering the strain energy due to thermal stresses resulting from the constrained motion of a body during a temperature change, the following equations are obtained;

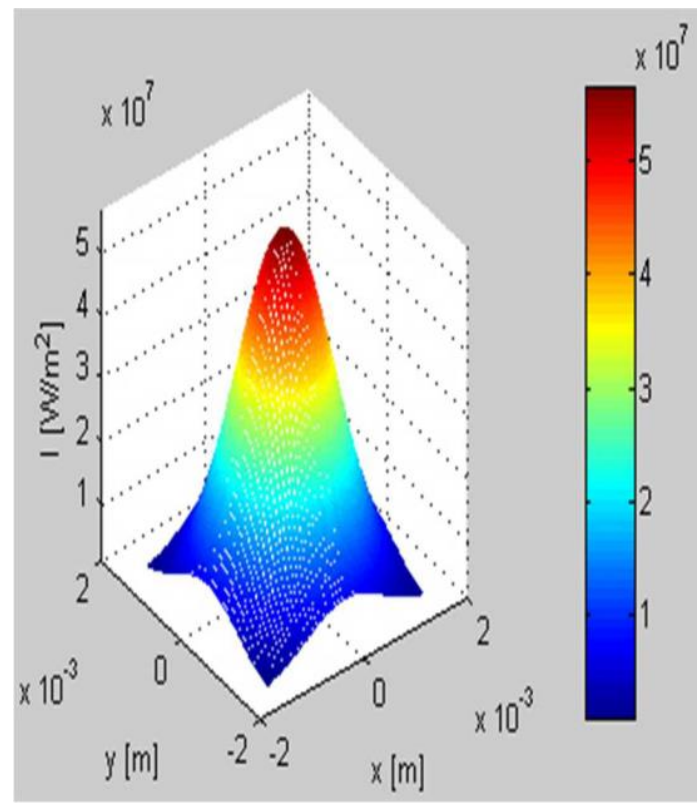

Fig.2. Gaussian distribution of a laser beam [7]

$\{\delta \mathrm{u}\}^{\mathrm{T} \int}[\mathrm{B}]^{\mathrm{T}}[\mathrm{B}][\mathrm{D}] \mathrm{dv}\{\mathrm{u}\}-\{\mathrm{du}\}^{\mathrm{T}} \int[\mathrm{B}]^{\mathrm{T}}[\mathrm{D}]\left\{\mathrm{e}^{\mathrm{th}}\right\} \mathrm{dv}=$ $\left.\{\delta \mathrm{u}\}^{\mathrm{T}} \int^{[\mathrm{Ns}}\right]^{\mathrm{T}}\{\mathrm{p}\} \mathrm{dS}+\{\delta \mathrm{u}\}^{\mathrm{T}}\left\{\mathrm{F}_{\mathrm{N}}\right\}$ 
ELK

Asia Pacific Journals

Noting that the $\{\delta \mathrm{u}\}^{\mathrm{T}}$ vector is a set of arbitrary virtual displacements common in all of the above terms. The above equation can be further reduced to

$$
[C(T)]\{T\}+[K(T)]\{T\}=\{Q(T)\}
$$

Where, $[\mathrm{K}]$ is the conductivity matrix and $[\mathrm{C}]$ is the specific heat matrix. $\{\mathrm{T}\}$ is the vector of nodal temperature, $K(T)$ is the temperature dependent stiffness matrix and $\{\mathrm{Q}\}$ is the nodal heat flow vector.

The finite element simulation is performed by using these parameters. The laser beam is moving along the $\mathrm{x}$-axis. The effect of mechanical deformation has not been considered during the analysis as it is negligible. ANSYS is used for analysis and determining the temperature distribution on the surface of the material. Thermal element SHELL157 is used for the transient heat flow analysis. A description of mesh is shown in the Fig. 3.

The simulation parameters taken for the analysis are given in the Table I.

TABLE I. THERMAL PROPERTIES OF TI-6AL-4V USED IN SIMULATION [5]

\begin{tabular}{|c|c|c|}
\hline $\begin{array}{c}\text { Temperature } \\
(\mathbf{K})\end{array}$ & $\begin{array}{c}\text { Thermal conductivity } \\
(\mathbf{W} / \mathbf{m K})\end{array}$ & $\begin{array}{c}\text { Specific heat } \\
(\mathbf{J} / \mathbf{k g K})\end{array}$ \\
\hline 373 & 30.5 & 300 \\
\hline 473 & 24.5 & 465 \\
\hline 673 & 20.4 & 551 \\
\hline 873 & 19.4 & 591 \\
\hline 1073 & 19.7 & 633 \\
\hline 1273 & 20.7 & 675 \\
\hline 1473 & 22.0 & 620 \\
\hline 1773 & 24.5 & 686 \\
\hline
\end{tabular}

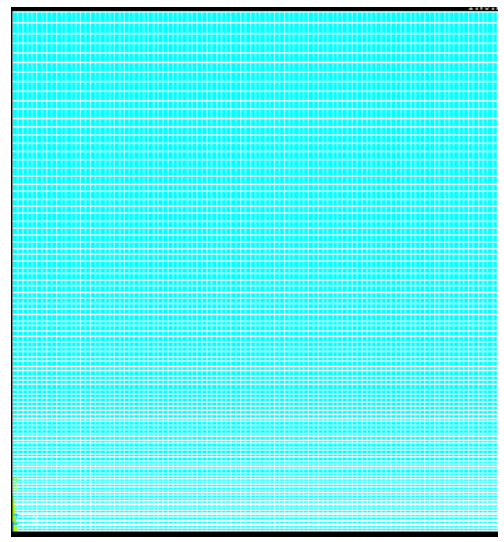

Fig.3. Two-dimensional finite element geometry and mesh.

\section{RESULTS AND DISCUSSIONS}

\section{ELK Asia Pacific Journals - Special Issue \\ ISBN: 978-81-930411-4-7}

The temperature distribution profile can be seen in the following figures at different time intervals. Fig.4(a) shows the position of the laser beam at time interval of 5 seconds. The distribution of the temperature profile obtained by the ansys analysis clearly shows that the point at which the laser is focussed has the maximum temperature value and the areas surrounding to it has low temperature values. Fig.4 (b) shows the position of the laser beam after 10 seconds. Now the laser beam has move further and the distribution of the profile obtained also shows that point at which the laser is focussed has the maximum temperature and the areas surrounding to it has low temperature value. Due to high variation in temperature the temperature gradient attains high values at the cut edges that results in high thermal stresses. The result clearly shows that as laser is moved along the surface of the material the temperature of the left surface decreases. The area where the laser is focussed reaches to the melting point of the material.

Fig.5 shows the variation of temperature with time over distance. We can see from the graph that as the distance is increased the temperature of the surface of the material decreases with time. The cooling happens due to radial conduction and convection from the surface.

\section{CONCLUSIONS}

A finite element modeling of laser cutting of Ti$6 \mathrm{Al}-4 \mathrm{~V}$ has been developed by using ANSYS finite element software. The numerical simulation results show that the temperature rises in the region where laser beam is focussed. The value of temperature gradient is high at the vicinity of the cut edges. As the beam further move on to the surface the corresponding surfaces experience a sharp decay in temperature. The sharp decay of temperature in the vicinity of the cut edge is associated

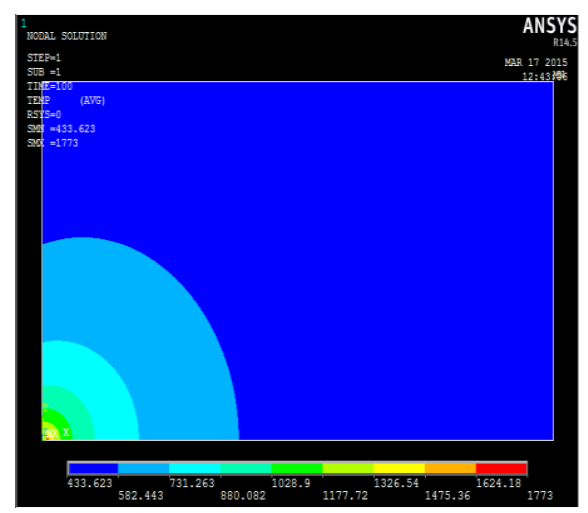

Fig.4. (a) Temperature distribution along the cutting edge at $t=5 \mathrm{sec}$ 
ELK

Asia Pacific Journals

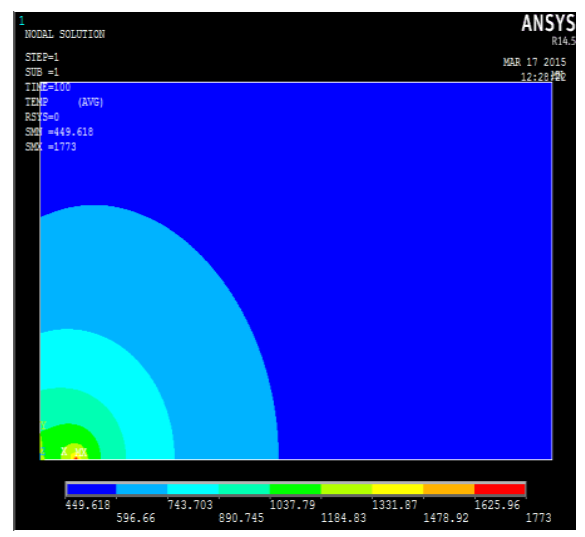

Fig.4. (b) Temperature distribution along the cutting edge at $\mathrm{t}=10 \mathrm{sec}$

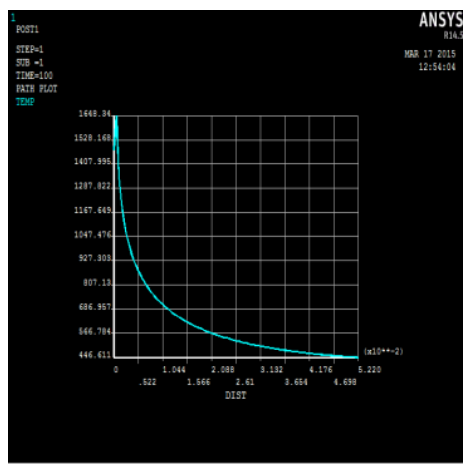

Fig.5. Time Temperature distribution curve

with heat conduction and convection phenomena, in which case, heat conduction accelerates energy transfer from the surface region to the solid bulk and convection transfers from the boundaries of the material. Titanium has low thermal conductivity, therefore the maximum temperature area is narrow.

\section{REFERENCES}

[1] V.K.Jain, Advanced Machining Processes, Allied Publisher, New Delhi, 2007, pp.186-206.

[2] J.D. Majumdar and I Manna, Laser Processing of Materials, Sadhana publications New Delhi, 2003, pp.495-562.

[3] A.K. Pandey and A.K. Dubey, "Modeling and optimization of Kerf taper and surface roughness in laser cutting of titanium alloy sheet",Journal of Mechanical Science and Technology, vol27, pp.1-10, 2013.

[4] A.K. Dubey and Vinod Yadava, "Laser beam machining-A review". International Journal of Machine Tools \& Manufacture, vol48, pp.609-628, 2008.

\section{ELK Asia Pacific Journals - Special Issue \\ ISBN: 978-81-930411-4-7}

[5] A.F.M. Arif and B.S. Yilbas, "Thermal Stress Developed During the Laser Cutting Process: Consideration of Different Materials", International Journal in Advanced Manufacturing Technology, vol37, pp.698-704, 2008,.

[6] K.Y. Nyon, C.Y. Nyeoh, Mohzani Mokhtar and R.A Rahman," Finite element analysis of laser inert gas cutting on Inconel 718", International Journal in Advanced Manufacturing Technology, vol60, pp.995-1007, 2012.

[7] Pawan Sharma, A.K. Dubey and A.K. Pandey, "Numerical Study of Temperature and Stress Fields in Laser Cutting of Aluminium Alloy Sheet", International Conference in Advances in Manufacturing and Material Engineering, vol5, pp.1887-1896, 2014.

[8] M. Sundar,P. T. Mativenga, Li L. and P. L.Crouse., "Laser removal of TiN from coated carbide substrate",International Journal of Advanced Manufacturing Technology, vol 4, pp.1169-1178, 2009.

[9] N.S.M. Shalahim, T.T. Mon, M.F. Ismail, M.F.F. Rashid and M.R.M. Rejab, "Finite Element Simulation of Laser-Micromachining". International Multi Conference of Engineers and Computer Sciences, held at Hong Kong,2010pp. 5-8.

[10] S.S Akhtar, "Laser Cutting of Thick-section Circular Blanks: Thermal Stress Prediction and Micro structural Analysis",International Journal of Advanced Manufacturing Technology, vol7, pp. 1345-1358, 2014.

[11] Syed Sohail Akhtar, "Laser Cutting of Thick-section Circular Blanks: Thermal Stress Prediction and Micro structural Analysis", International Journal of Advanced Manufacturing Technology, vol 71, pp.1345-1358, 2014.

[12] B.S. Yilbas, "Laser cutting of rectangular geometry into alumina tiles". International Journal in Advanced Manufacturing Technology, vol 54,pp.3543, 2013.

[13] R .Akarapu, B.Q. Li, and A. Segall, "A thermal stress and failure model for laser cutting and forming operations, J Failure Anal Prevent, vol 5, pp.51-62, 2004

[14] Salman Nisar, M. A.Sheikh, Li Lin and A.J.Pinkerton, "The Effect of Laser Beam Geometry on Cut Path Deviation in Diode Laser Chip-Free Cutting of Glass". Journal of Manufacturing Science and Engineering, vol 132, pp.1-9, 2012.

[15] B.S. Yilbas, S.S. Akhtar, and C. Karatas, "Laser straight cutting of zirconia tiles", Journal of Mechanical Science and Technology, vol 26, pp. 591-599, 2012. 
[16] S.Z. Shuja and B.S. Yilbas, "Laser Multi-Beam Heating of Moving Steel Sheet: Thermal Stress Analysis", Optics and Laser in Engineering, vol51, pp.446-452, 2013.

[17] Y. Qui, Y. Chen, X. Ni, Z. Shen, J. Bi, and X. Zhang, "Axisymmetric Numerical Simulation of Plastic Damage in Aluminium Alloys Induced by long Pulsed Laser", Optics and Lasers in Engineering, vol48, pp.361-367, 2010.

[18] M.S.Gross,I. Black, and W.H.Muller, "3-D simulation model forgas-assisted laser cutting", Lasers Eng vol 15, pp.129-146, 2005.

[19] S.V. Shalupaev, E.B. Shershnev, Y.V. Nikityuk and A.A. Sereda, "Two-beam laser thermal cleavage of brittle nonmetallic materials", Optics and Lasers In Engineering, vol 73, pp.356-359, 2006.

[20] A.K. Pandey and A.K. Dubey, "Simultaneous optimization of multiple quality characteristics in laser cutting of titanium alloy sheet", Optics and Laser Technology, vol 56, pp.1-7, 2012.

[21] A.K. Pandey and A.K. Dubey, "Fuzzy Expert System for Prediction of Kerf Qualities in Pulsed Laser Cutting of Titanium Alloy Sheet". Machining Science and Technology, vol 17, pp. 545-574, 2013. 